\title{
Remote Detection of Chemicals by Millimeter-Wave Spectroscopy*
}

$$
\begin{aligned}
& \text { ANL/ET/CP-97111 } \\
& \text { CONF-980731- }
\end{aligned}
$$

by

N. Gopalsami and A. C. Raptis

Energy Technology Division

Argonne National Laboratory

Argonne, IL 60439

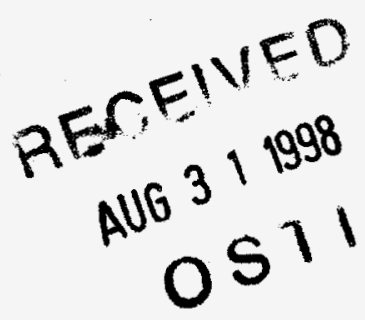

The submitted manuscript has been authored by a contractor of the U.S. Government under contract No. W-31-109-ENG-38. Accordingly, the U.S. Government retains a nonexclusive, royalty-free license to publish or reproduce the published form of this contribution, or allow others to do so, for U.S. Government purposes.

Manuscript submitted to SPIE'98, 4th International Conference on Millimeter and Submillimeter Waves and Applications, 20-23 July 1998, San Diego, CA.

*This work is supported by the U.S. Department of Energy, Office of Nonproliferation and National Security, Office of Research and Development, under Contract W-31-109ENG-38.

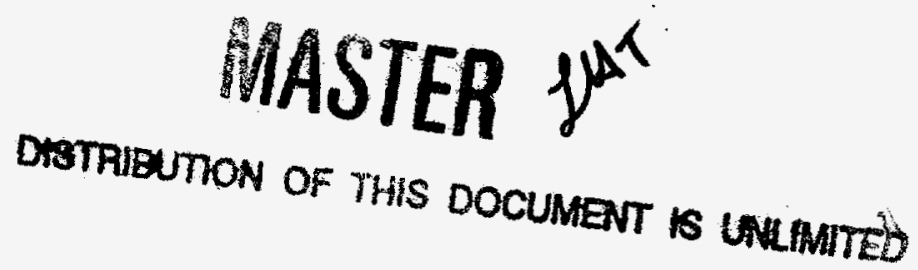




\section{DISCLAIMER}

This report was prepared as an account of work sponsored by an agency of the United States Government. Neither the United States Government nor any agency thereof, nor any of their employees, makes any warranty, express or implied, or assumes any legal liability or responsibility for the accuracy, compieteness, or usefulness of any information, apparatus, product, or process disclosed, or represents that its use would not infringe privately owned rights. Reference herein to any specific commercial product, process, or service by trade name, trademark, manufacturer, or otherwise does not necessarily constitute or imply its endorsement, recommendation, or favoring by the United States Government or any agency thereof. The views and opinions of authors expressed herein do not necessarily state or reflect those of the United States Government or any agency thereof. 


\section{DISCLAIMER}

Portions of this document may be illegible in electronic image products. Images are produced from the best available original document. 
Remote Detection of Chemicals by Millimeter-Wave Spectroscopy

\author{
N. Gopalsami and A. C. Raptis \\ Energy Technology Division \\ Argonne National Laboratory, Argonne, Illinois 60439
}

\begin{abstract}
This paper discusses the development and field testing of a remote chemical detection system that is based on millimeter-wave (mm-wave) spectroscopy. The mm-wave system is a monostatic sweptfrequency radar that consists of a mm-wave sweeper, a hot-electron-bolometer detector, and a trihedral reflector. The chemical plume to be detected is situated between the transmitter/detector and the reflector. Millimeter-wave absorption spectra of chemicals in the plume are determined by measuring the swept-frequency radar return signals with and without the plume in the beam path. The problem of pressure broadening, which hampered open-path spectroscopy in the past, has been mitigated in this work by designing a fast sweeping source over a broad frequency range. The heart of the system is a Russian backward-wave oscillator (BWO) tube that can be tuned over 225-315 GHz. A mm-wave sweeper that includes the BWO tube was built to sweep the entire frequency range within $10 \mathrm{~ms}$. The radar system was field-tested at the DOE Nevada Test Site at a standoff distance of $60 \mathrm{~m}$. Methyl chloride was released from a wind tunnel that produced a $2-\mathrm{m}$ diameter plume at its exit point. The mmwave system detected methyl chloride plumes down to a concentration of $12 \mathrm{ppm}$. The measurement results agree well with model-fitted data.
\end{abstract}

Key Words: Millimeter waves, backward-wave oscillator, molecular spectroscopy, standoff monitoring.

\title{
1 INTRODUCTION
}

Remote or standoff sensing of airborne chemicals is gaining importance for arms control and treaty verification, intelligence collection, and environmental monitoring. When compared with point detection or sampling techniques, the remote method is nonintrusive and can be used to monitor wide areas. Optical and laser techniques for sensing volatile organics and other hazardous chemicals are under active development in the infrared, visible, and ultraviolet regions of the electromagnetic spectrum. ${ }^{1-2}$ The purpose of this work is to investigate the use of the mm-wave spectrum to remotely monitor chemicals. The mm-wave sensors use longer wavelengths than those of optics and thus can operate under all weather conditions and in smoky and dusty environments.

Millimeter-wave spectroscopy is a well-established laboratory technique for determining the structure and dynamics of molecules in the gas or vapor phase. ${ }^{3}$ Millimeter-wave spectral lines are narrow and nearly $100 \%$ specific when analyzed in a gas cell under low pressures $(<1$ torr). However, when applied to open-air monitoring, the higher pressures cause the spectral lines to broaden because of molecular collisions. A typical air-broadening parameter of a molecule is $4 \mathrm{MHz} /$ torr (half width at half height) and it is $\approx 4 \mathrm{GHz}$ at $1 \mathrm{~atm}$. Conventional detection methods that are capable of high detection 
sensitivities at low pressures, e.g., derivative or phase lock techniques, are not directly applicable to open-air detection. Hence, new detection methods and systems are needed for remote detection of trace chemicals in air.

One way to overcome the pressure-broadening effect is to use a swept-frequency mm-wave source that can sweep over a broad frequency range in a short time. The wide-frequency sweep improves spectral line resolution and molecular selectivity, whereas the fast sweep allows for efficient baseline subtraction by direct video detection. The feasibility of open-air mm-wave spectroscopy was first demonstrated by using a monostatic, swept-frequency radar system in the $225-315 \mathrm{GHz}$ range. ${ }^{4}$ The $\mathrm{mm}$ waves were generated with a low-frequency sweeper and low-power frequency triplers. The narrow bandwidth of the triplers also limited the tuning range to within $50 \mathrm{GHz}$. The proof-of-principle of this system was tested by detecting $\mathrm{D}_{2} \mathrm{O}$ in open air. In addition, the chemical selectivity of the technique was demonstrated by using a special deconvolution procedure. ${ }^{5}$ Individual chemicals in a multicomponent mixture were determined from both simulated and experimental spectra.

With recently available backward-wave-oscillator (BWO) tubes (Model OB-30) from Istok Corporation, Russia, we developed a prototype radar system that can sweep the entire frequency range within $10 \mathrm{~ms}$ and generate up to $10 \mathrm{~mW}$ of power. ${ }^{6}$ This higher power allows a longer detection range and the wider tuning range improves the resolution of spectral lines. The radar system was recently field-tested at the DOE Nevada Test Site at a standoff distance of $60 \mathrm{~m}$. This paper describes the mmwave sensor, the field test setup, and test results for methyl chloride releases.

\section{DESCRIPTION OF THE MM-WAVE SENSOR}

The mm-wave sensor, shown in Fig. 1, is a monostatic radar system that operates in an atmospheric window in the frequency range of $225-315 \mathrm{GHz}$. Swept-frequency signals in the $225-315 \mathrm{GHz}$ range are generated by a mm-wave sweeper and transmitted through a chemical plume. A corner cube, located behind the plume, returns the radar signals through the plume to a mm-wave detector situated next to the transmitter. Millimeter-wave absorption spectra of chemicals in the plume are determined by measuring swept-frequency radar signals with and without the plume in the beam path. A Fabry-Perot (F-P) cavity, that consumes a small part of the mm-wave energy, generates frequency markers for on-the-fly calibration of frequencies. ${ }^{7}$

The mm-wave sweeper includes BWO tube (OB-30) from the Istok Company, Russia. The tube, 25 $\mathrm{mm}$ in diameter and $35 \mathrm{~mm}$ long, is water cooled. It requires an AC filament power supply (6.4 V, 1.35 A) to the cathode heater and a high-voltage (HV) power supply $(1000-4000 \mathrm{~V}, 40 \mathrm{~mA})$ between the cathode and the slow-wave structure to accelerate electrons. A DC magnetic field of $7000 \mathrm{G}$ is required to steer the electron beam over the slow-wave structure. A permanent magnet made of cobalt-samarium alloy produces the magnetic field. To align the tube properly with respect to the DC magnetic field, which is necessary to generate $\mathrm{mm}$-wave oscillations, it is mounted in the pole gap of the magnet with a three-axis alignment fixture. The tube, tunable from 225 to $350 \mathrm{GHz}$ by varying the anode (slow-wave 
structure) voltage from 1000 to $4000 \mathrm{~V}$, provides an average of $10 \mathrm{~mW}$ power. The radiation is coupled to a scalar horn through an over-moded rectangular waveguide that is attached to the tube structure.

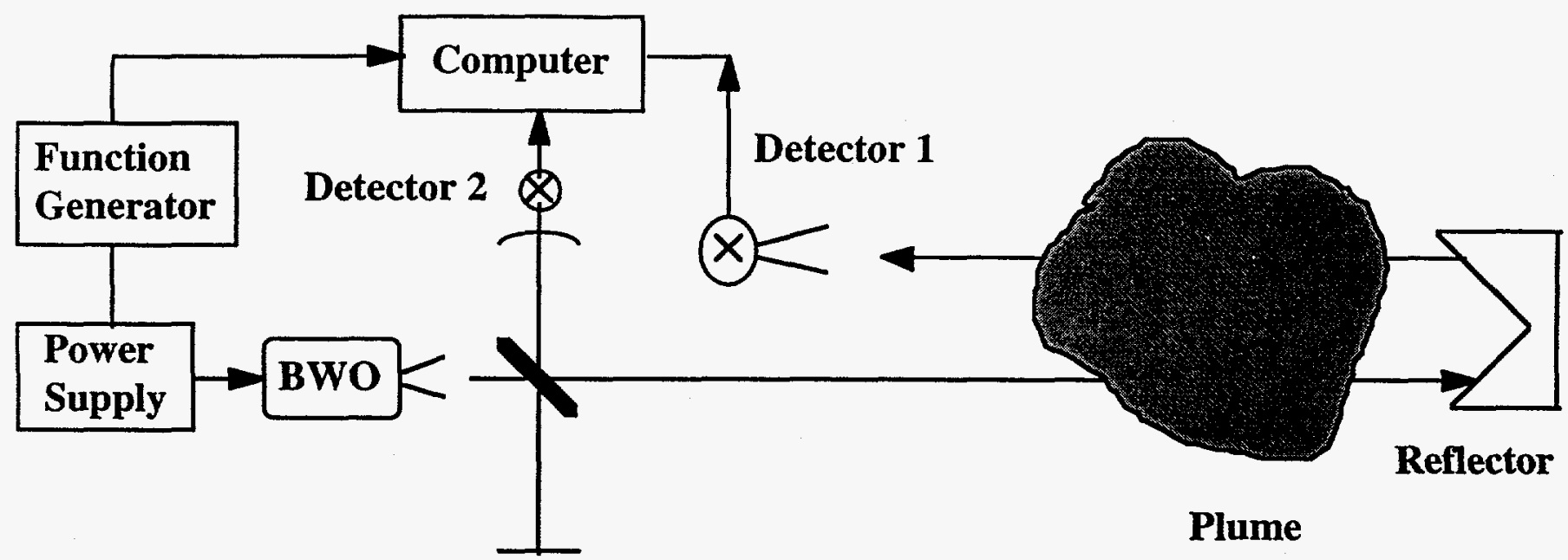

Fabry-Perot Cavity

Fig. 1. Schematic diagram of mm-wave radar system

The stability of mm-wave radiation generated by the BWO tube is affected by fluctuations in the voltage of the power supply, misalignment of the tube within an applied external magnetic field, and temperature of the tube structure. Because the BWO tube has an extremely low internal capacitance, all HV noise, including $60-\mathrm{Hz}$ ripple and switching power-supply noise $(20 \mathrm{kHz})$, are converted into frequency variation. Three types of instabilities (blinking effect, mode breaks, and mode switching) may occur while the tube is being tuned, but in most cases these instabilities can be eliminated by proper alignment of the tube within the magnet. To control the temperature of the tube structure and thus ensure long-term stability of the radiation, the tube is water-cooled by a temperature-controlled closedcycle water pump.

Despite all of the precautions one may take to control BWO stability, it is nearly impossible to achieve the frequency stability required for baseline subtraction and signal averaging. Hence, one often resorts to phase-locked control of the BWO in high-resolution spectroscopy, but this is rather slow for the wide-frequency sweep required in open-path spectroscopy. In this work, we use a quasi optical F-P cavity for on-the-fly calibration of frequencies. The F-P cavity is formed between a movable spherical mirror and a plane mirror. A beam splitter couples part of the main-beam radiation into the cavity. The radiation that is transmitted directly through the beam splitter is used to measure the spectrum of the gaseous plume. The cavity power, coupled out of the cavity by an iris opening in the plane mirror, is detected by a Schottky detector.

When swept-frequency radiation is applied to the cavity, resonant peaks are generated each time the cavity spacing is equal to the integral number of half-wavelengths. For a cavity length of $d$ between the 
resonating mirrors, the marker spacing in frequency is given by $\mathrm{c} / 2 \mathrm{~d}$, where $\mathrm{c}$ is the wave velocity in free space. Data that correspond to radar signal and cavity markers are collected during each sweep. Because the cavity markers correspond to actual frequencies during a sweep, the cavity markers and the corresponding samples of radar data are aligned with respect to a reference data set by computer software. The radar data between the cavity markers are interpolated linearly. Thus, the use of an F-P cavity enables us to calibrate, on the fly, the frequencies of a free-running oscillator, which leads to excellent baseline subtraction and signal averaging.

\section{FIELD TEST SETUP}

Using a wind tunnel at the Nevada Test Site at Frenchman Flat near Mercury, Nevada, we field tested the mm-wave system during May 12-15, 1997. The wind tunnel is capable of producing wellcharacterized open-air plumes with desired concentrations of chemical vapors. Chemicals are injected into the air stream of the wind tunnel and atomized with nozzles. Fans outside the wind tunnel produce high speed turbulent air that carries the injected chemicals into a well mixed plume. The flow rate of the air stream is set sufficiently high so the shape of the exhaust plume is not affected by the ambient wind for up to a few meters from the exit point of the wind tunnel. The plume diameter at the wind tunnel exhaust is $2 \mathrm{~m}$. Chemical is delivered to the nozzle manifold either by pressurization of the chemical storage tank, with nitrogen in the case of liquids, or by the self-pressure of gases in the case of compressed gases or compressed liquefied gases. Flow rate of the chemicals injected into the nozzles is controlled and monitored remotely from the control room. Concentrations of released chemicals are determined from the mass flow rate measurements of injected chemicals and air flow rate. The release concentrations can be controlled from a few parts per million by volume (ppmV) to $1200 \mathrm{ppmV}$.

The test of the mm-wave sensor was staged from a trailer $60 \mathrm{~m}$ away from the wind tunnel, in the cross wind direction. The trailer was fitted with an air conditioner and 110-V single-phase power supply. Figure 2 is a picture of the $\mathrm{mm}$-wave sensor inside the trailer. The mm-wave radiation $(\approx 10$ $\mathrm{mW}$ of power) was transmitted through one of the trailer windows into the wind tunnel plume. The window was covered with a plastic film that was transparent to $\mathrm{mm}$ waves. A 6-in. lens, focused at infinity, was used to provide a highly directed beam. The cone angle of the beam is $0.37^{\circ}$, with a footprint of $0.6 \mathrm{~m}$ at the wind tunnel. An aluminum corner cube with a $0.9 \times 0.9 \times 0.9-\mathrm{m}$ opening was fabricated and mounted behind the plume to return radar signals. The return signals were collected by a 6-in. lens, coupled to a liquid-helium-cooled (hot electron) bolometer detector situated next to the transmitter. Figure 3 shows the mm-wave sensor trailer together with the wind tunnel and corner cube.

For safety reasons, the trailer was not manned during the release, leaving the mm-wave sensor unattended during the test. Typically, an area sweep occurred at $\approx 11: 30 \mathrm{AM}$ at which time the final checks on the sensor were made and trailer was evacuated. Data were collected remotely from the windtunnel control room, $1.7 \mathrm{~km}$ away, via an $\mathrm{RF}$ link between a master computer in the control room and the data-acquisition (slave) computer in the trailer in master/slave fashion. The trailer computer was hardwired for a hard re-boot from the control room in the event of a computer crash. 


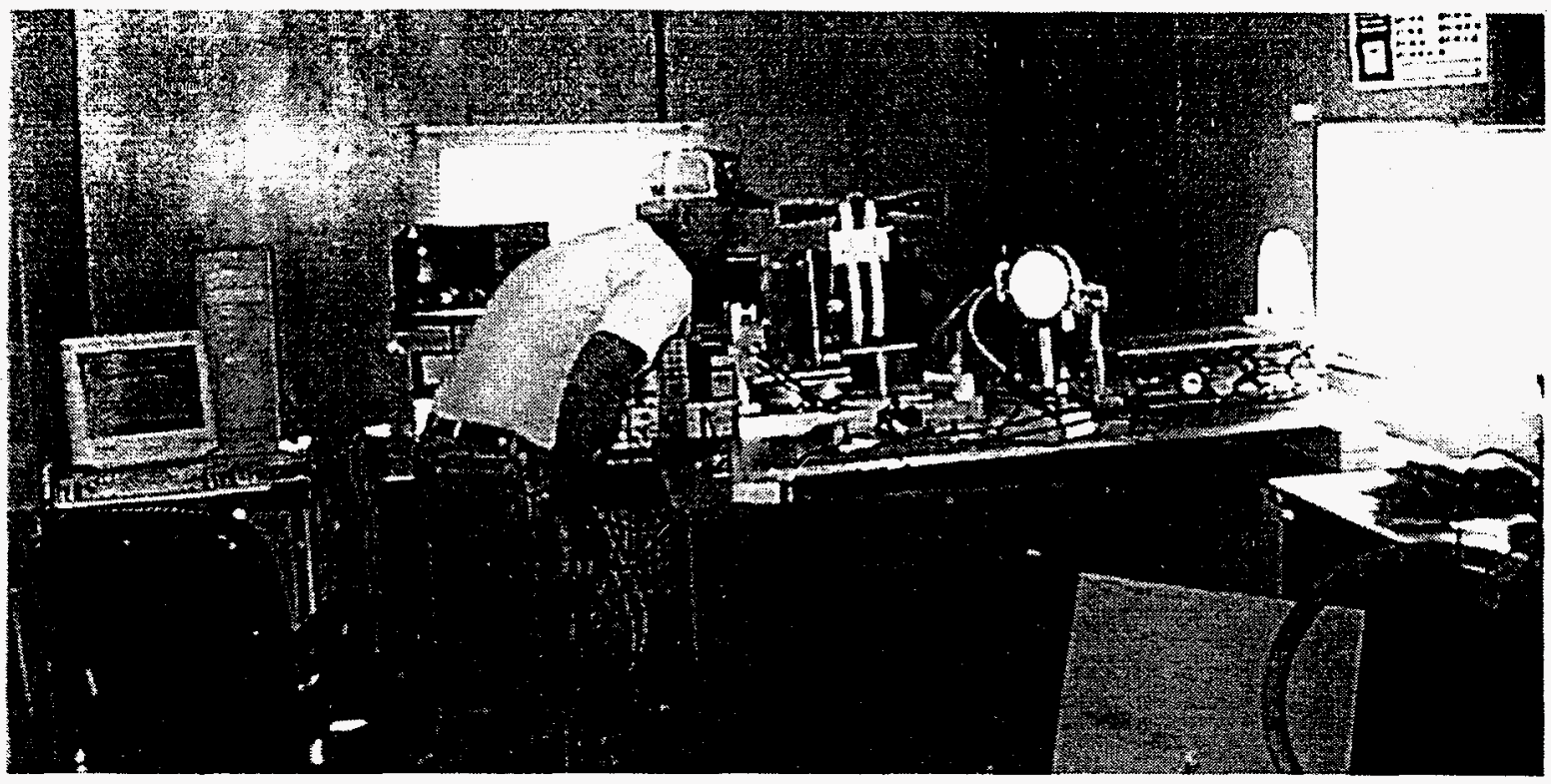

Fig. 2. Millimeter-wave sensor antenna being aligned with corner cube

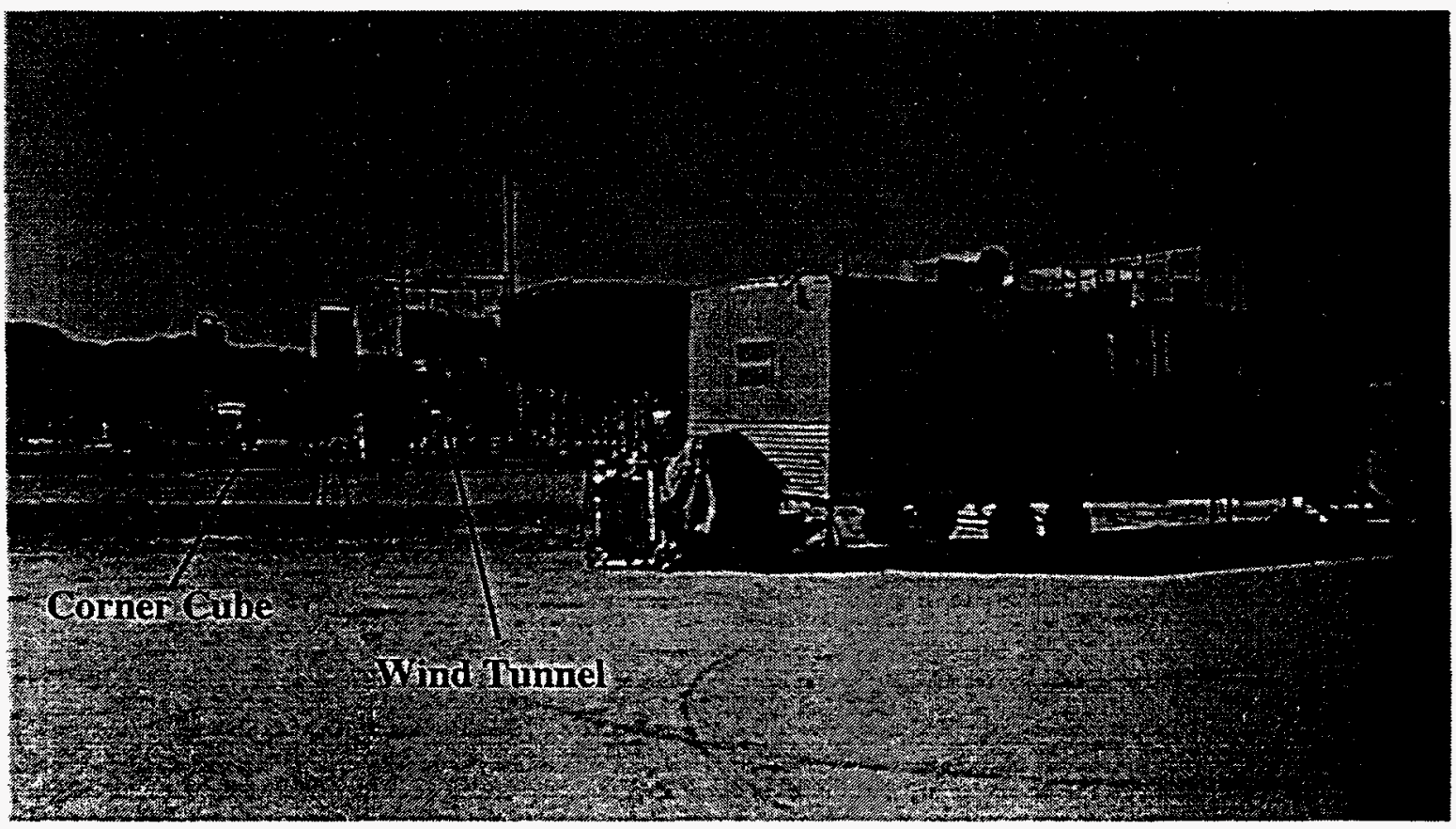

Fig. 3. Millimeter wave system trailer, wind tunnel, and corner cube 


\section{TEST RESULTS}

The mm-wave spectral data of methyl chloride were first measured in the laboratory with a 1.4-m gas cell. To simulate atmospheric pressure, 377 mtorr of methyl chloride was mixed with nitrogen to a pressure of $1 \mathrm{~atm}$. Figure 4 shows the measured and model absorption lines in the frequency range of 230-298 GHz. The model lines were simulated with data from the JPL molecular database. ${ }^{8}$ Because of the natural abundance of $\mathrm{Cl}^{35}$ and $\mathrm{Cl}^{37}$ in a 3:1 ratio, methyl chloride exhibits three pairs of distinct spectral lines (at 237, 239; 262, 265; and 288, $292 \mathrm{GHz}$ ) when measured at low pressure. At atmospheric pressure, $\mathrm{Cl}^{35}$ and $\mathrm{Cl}^{37}$ lines overlap, as shown in Fig. 4, but their presence is revealed by the slope changes in the absorption curve.

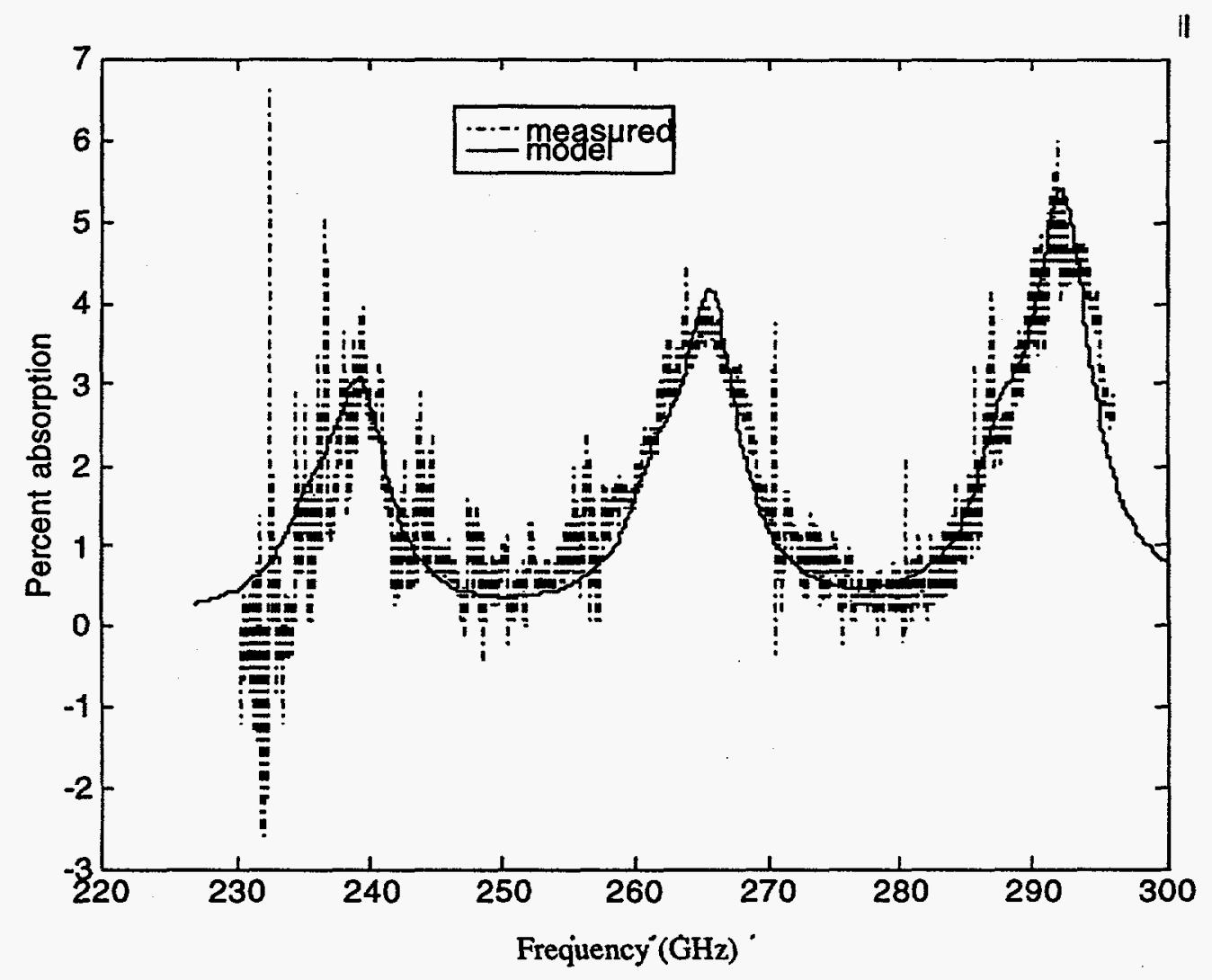

Fig. 4. Methyl chloride spectra obtained in the laboratory from $377 \mathrm{mtorr}$ of methyl chloride mixed with nitrogen to $1 \mathrm{~atm}$ in $1.4-\mathrm{m}$ gas cell

The data acquisition procedure for the field test consisted of a frequency sweep of $226-298 \mathrm{GHz}$ in $20 \mathrm{~ms}$, with 100 averages. Before the tests, the system frequencies were calibrated with a gas cell and acetonitrile. The release profile of methyl chloride was in the form of an on-off pattern, each pattern typically lasting $\approx 5 \mathrm{~min}$. Reference and plume signals were taken during the plume-off and -on periods, respectively. Three channels of data, 10,000 samples per channel, were collected each time: DC- and AC-coupled data from the bolometer and AC-coupled F-P cavity data. The AC-coupled channels 
worked well, but the DC-coupled channel drifted slowly, and eventually saturated $\approx 2 \mathrm{~h}$ after the start of each day of test. To interpret the spectral data, we will next develop the data analysis procedures for the $\mathrm{DC}$ - and AC-coupled cases.

\section{DC-coupled data}

The DC-coupled data collection refers to measurement of the absolute power of mm waves with respect to frequency. Let $P_{0}(v)$ be the output power of the transmitter as a function of frequency, and $P_{1}(v)$ and $P_{2}(v)$ the power measured by the detector without and with the plume, called reference and plume signals, respectively. Then

$$
P_{1}(v)=P_{0}(v) T
$$

and

$$
P_{2}(v)=P_{0}(v) T \exp [-\alpha(v) C \ell]
$$

where $T$ is the two-way transmission coefficient of the atmosphere between the transmitter antenna and receiver, $\alpha(v)$ is the absorption coefficient $\left(\mathrm{cm}^{-1}\right)$ of $1 \mathrm{ppm}$ of chemical in the air at frequency $v, C$ is the concentration of the chemical (ppm), and $\ell$ is the path length $(\mathrm{cm})$ of the plume. The difference between the reference and plume signals $D(v)$ is given by

$$
D(v)=P_{1}(v)-P_{2}(v)=P_{1}(v)\{1-\exp [-\alpha(v) C \ell]\} .
$$

Thus, in the DC-coupled case, the absorption coefficient $\alpha(v)$ can be determined by dividing Eq. 3 by the reference signal. For trace amounts of gases to be released in the open air, $\alpha(v) C \ell$ is small, and Eq. 3 becomes

$$
D(v)=\alpha(v) C \ell P_{1}(v)
$$

\section{$\underline{\text { AC-coupled data }}$}

The AC-coupled data refers to the ac-type signal (high-pass filtered signal) from the detector circuit that blocks the DC-level signals. This mode of data collection avoids saturation of the detector preamplifier if the detector signal drifts with time. Let a bar over the variables represent AC-coupled signal operation. For the AC-coupled case, Eqs. 1 and 2 become

$$
\bar{P}_{1}(v)=\bar{P}_{0}(v) T
$$

and

$$
\overline{\mathrm{P}}_{2}(v)=\overline{\mathrm{P}}_{0}(v) \mathrm{T}-\overline{\mathrm{P}_{0}(v) \mathrm{T} \alpha(v) \mathrm{C} \ell}+\frac{\overline{\mathrm{P}_{0}(v) \mathrm{T}\{\alpha(v) \mathrm{C} \ell\}^{2}}}{2}-\ldots
$$

For gaseous absorption lines that are small and broad, the difference between the reference and plume signals, Eq. 5 - Eq. 6, becomes 


$$
\overline{\mathrm{D}}(v)=\overline{\mathrm{P}}_{1}(v)-\overline{\mathrm{P}}_{2}(v)={\overline{\mathrm{P}_{0}(v) \alpha(v)}}^{\mathrm{C}} \mathrm{C} \ell \mathrm{T} \cong \overline{\mathrm{P}}_{1}(v) \alpha(v) \mathrm{C} \ell .
$$

Thus, the difference signal, (Eq. 7), represents an amplitude modulation of the reference signal by the plume absorption coefficient. As in Eq. 3 of the DC-coupled case, it is not possible to separate out the absorption coefficient by dividing the difference signal by $\overline{\mathrm{P}}_{0}(v)$ because of its $\mathrm{AC}$ nature and possible zero crossings. However, the absorption coefficient $\alpha_{m}(v)$ of a chemical can be determined from one of the published molecular data bases, such as the JPL catalog, or by measuring it with a gas-cell spectrometer. In that case, $\bar{D}(v)$ in Eq. 7 can be fitted with a model $\bar{E}(v)$, given by

$$
\overline{\mathrm{E}}(v)=\alpha_{\mathrm{m}}(v) \mathrm{C} \ell \overline{\mathrm{P}}_{1}(v),
$$

where the constant $\mathrm{C}$ may be chosen such that it minimizes the values of the integral of the squared error, $S(C)$, over the sweep frequencies $f_{1}$ to $f_{2}$. Thus,

$$
S(C)=\int_{f_{1}}^{f_{2}}\left[\alpha_{m}(v) C \ell \bar{P}_{1}(v)-\bar{P}_{1}(v)+\bar{P}_{2}(v)\right]^{2} d v
$$

By setting $\mathrm{dS}(\mathrm{C}) / \mathrm{dC}=0$, the equation for $\mathrm{C}$ becomes

$$
C=\frac{\int_{f_{1}}^{f_{2}} \alpha_{m}(v) \ell \bar{P}_{1}(v)\left[\bar{P}_{1}(v)-\bar{P}_{2}(v)\right] d v}{\int_{f_{1}}^{f_{2}}\left[\alpha_{m}(v) \ell \bar{P}_{1}(v)\right]^{2} d v} .
$$

Figure 5 gives the AC-coupled data that correspond to $150 \mathrm{ppm}$ of methyl chloride, for example, in which the dotted line represents the difference signal between the reference and the plume, and the solid line represents the model-fitted signal. The model fits the measured data well, eliminating the high frequency noise. To show that the difference signal is an amplitude modulation of the reference signal by the plume absorption coefficient, we have overlain an envelope of appropriately scaled model absorption curves. 


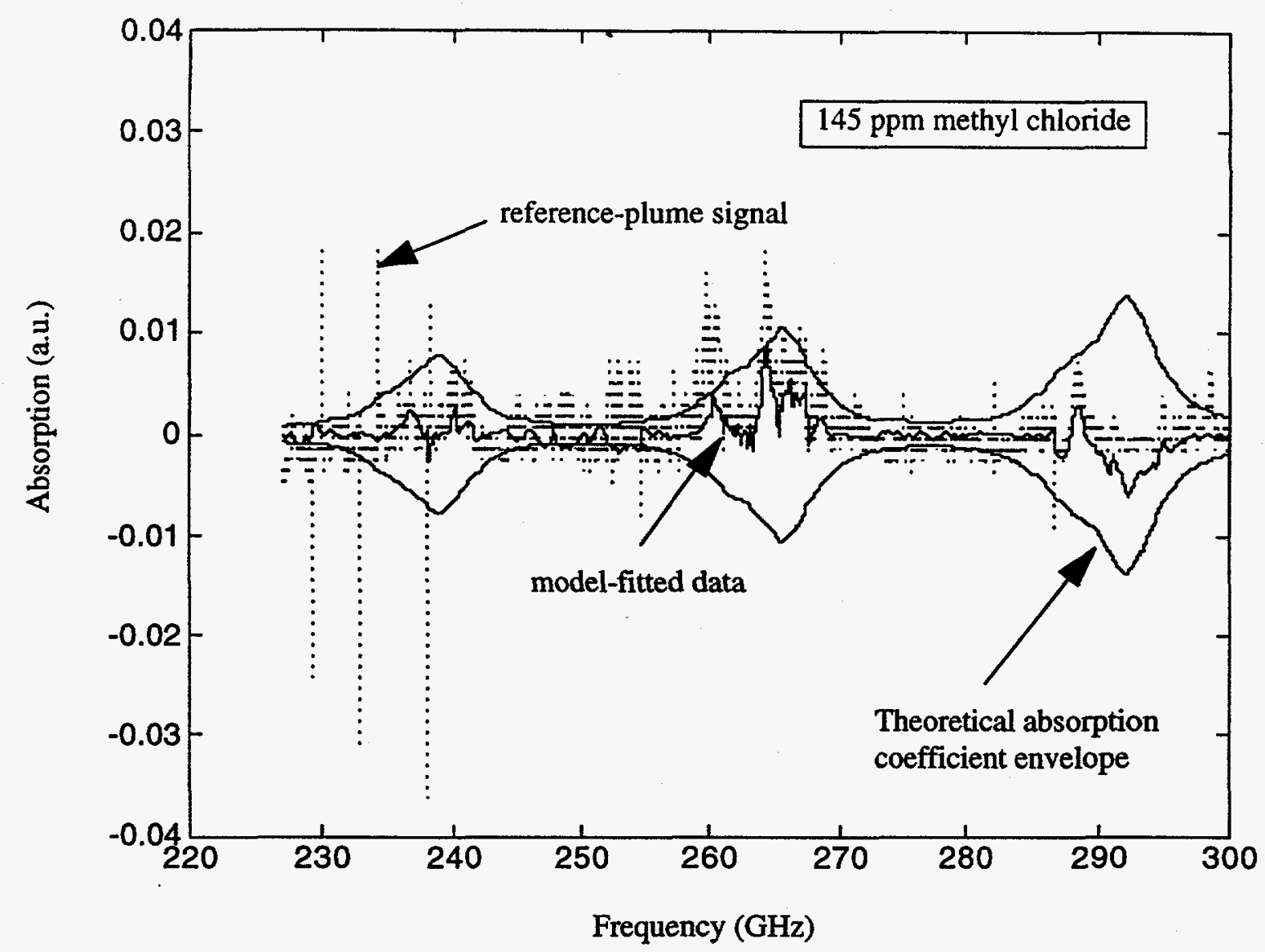

Fig. 5. Difference signal between the reference and the plume (dotted line), model-fitted signal (solid line), and absorption coefficient envelope (solid line)

Figure 6 presents plots of the model-fitted data for $12,45,145,318,370$, and $500 \mathrm{ppm}$ concentrations of methyl chloride. These model data agreed well with the corresponding difference data between the reference and the plume. Below $12 \mathrm{ppm}$, however, the correlation between the model and the difference data was found to be poor. The corresponding values of C, from Eq. 10, are plotted in Fig. 7 , with respect to the actual release concentrations. The value of $\mathrm{C}$ calculated from the model thus provides a good estimate of the release concentration. 


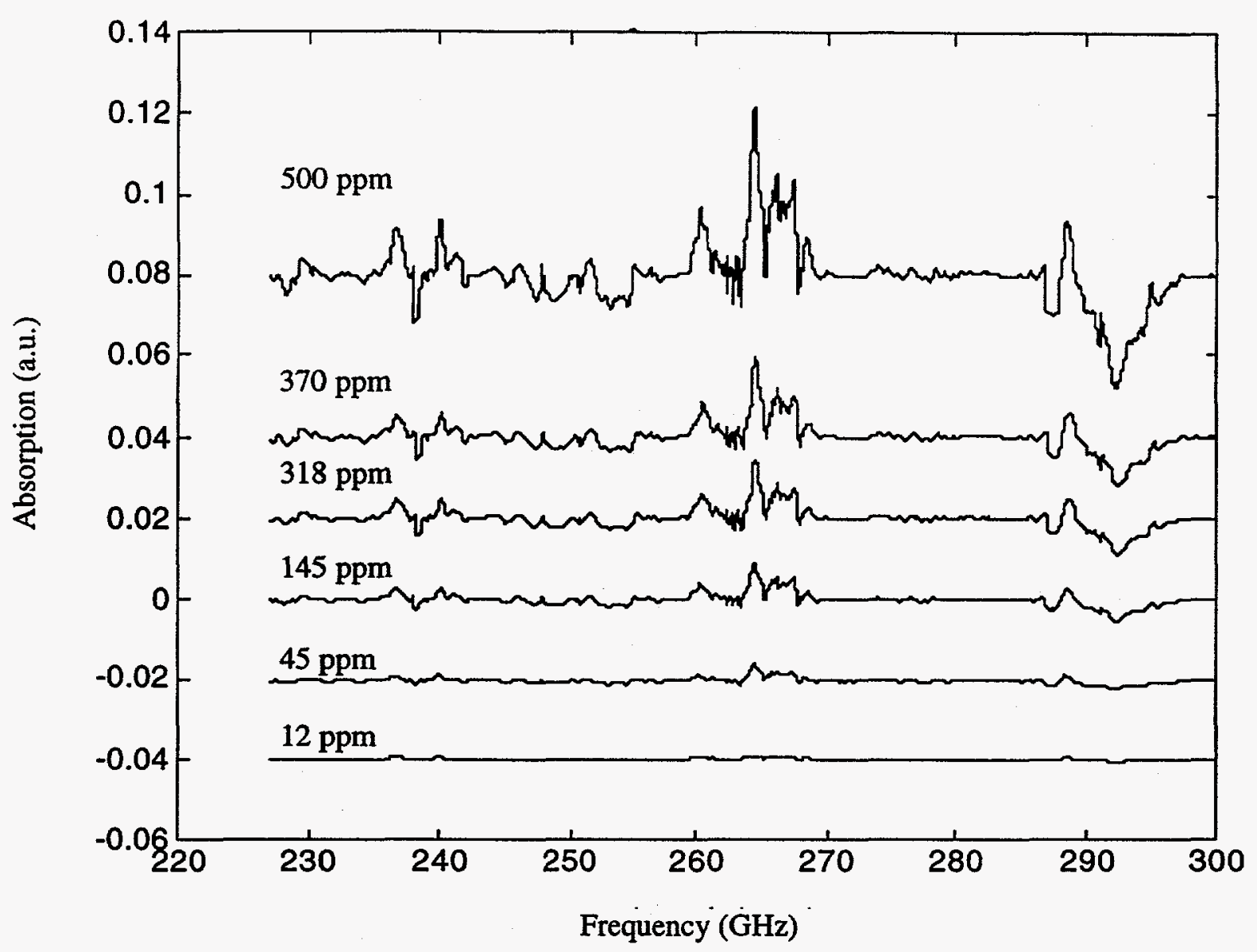

Fig. 6. Plots of model-fitted spectra for various concentrations of methyl chloride

\section{CONCLUSIONS}

A millimeter-wave chemical sensor, developed at Argonne National Laboratory, was field tested at the Nevada Test Site during May 12-15, 1997. The objective of the test was to demonstrate the capability of the sensor to remotely detect chemicals that are pertinent to verification of the arms control treaty and environmental monitoring. The test of the sensor was staged from a trailer at a standoff distance of $60 \mathrm{~m}$ from the plume. The return of radar signals was provided by a $0.9-\mathrm{m}$ corner cube mounted behind the plume.

Both DC- and AC-coupled bolometer data were collected remotely from a control room. Reference and plume signals were collected by using 20 -ms sweeps and 100 averages, with the plume on and off for 2 to $5 \mathrm{~min}$ intervals. Whereas the DC-coupled signals saturated the preamplifier $\approx 2 \mathrm{~h}$ after the start of the experiments, the AC-coupled mode of data collection worked well during the entire test period of $\approx 7 \mathrm{~h}$. The meteorological conditions during the tests were somewhat benign: $35^{\circ} \mathrm{C}, 8 \%$ relative humidity, and winds of $5-10 \mathrm{~m} / \mathrm{s}$. At times, wind gusts produced a blowing sand storm. 


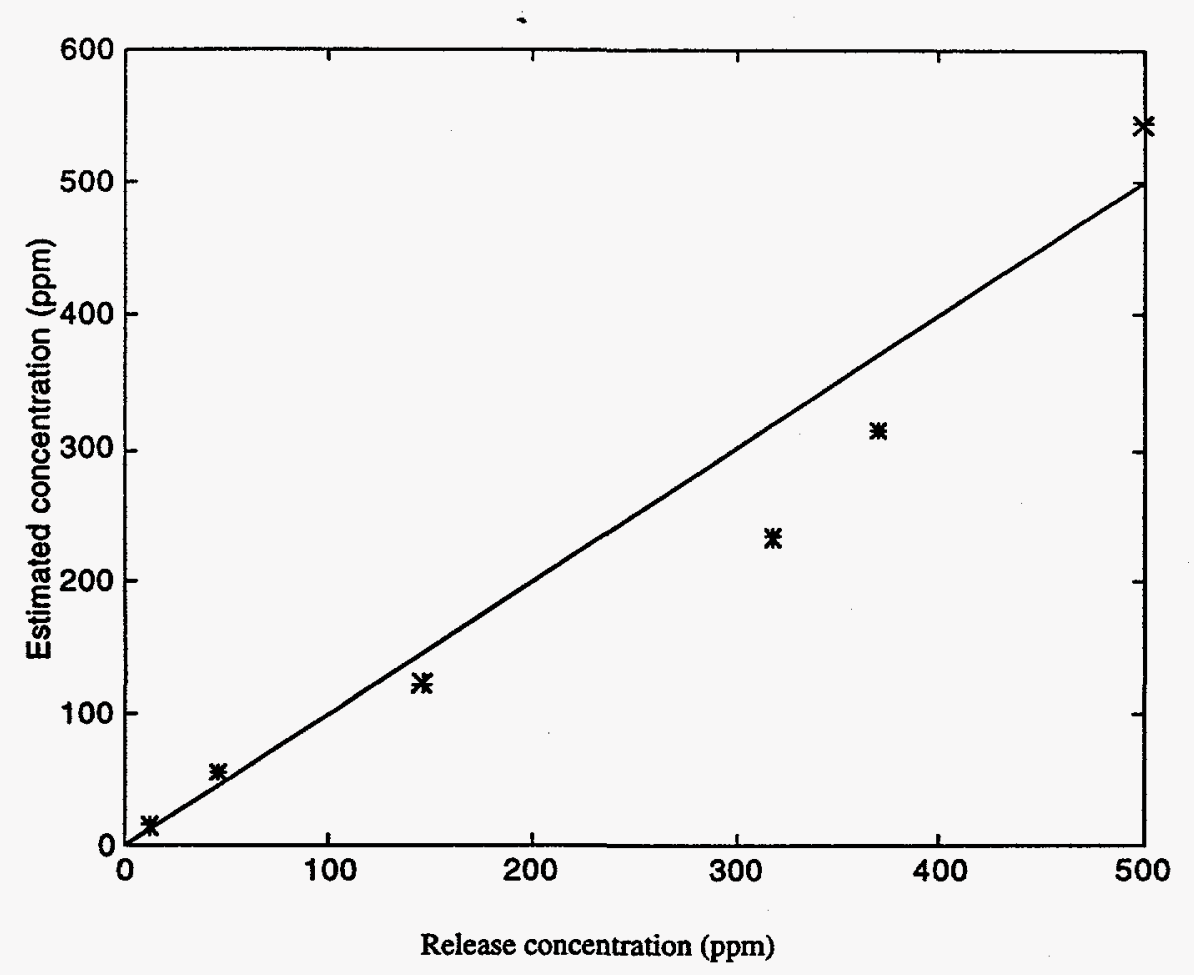

Fig. 7. Estimated concentrations based on model fit.

A model-based analysis procedure has been developed to interpret and quantify the acquired ACcoupled data. The analysis shows that the difference between the reference and plume signals is essentially an amplitude modulation of the reference signal by the plume absorption function. The correlation constant between the measurement and model data provides a quantitative estimate of the chemical concentration. Good agreement between the measured and model data was obtained for various release concentrations of methyl chloride, down to $12 \mathrm{ppm}$. A linear fit was obtained between the estimated and actual concentrations.

Because of generally poor long-term stability of the mm-wave sweepers, it was necessary to keep the time interval between the reference and plume signals short, within $\approx 2$ to $3 \mathrm{~min}$, to obtain reproducible results. One approach to further improve the detection sensitivity is to use two reflectors, one in the front and another in the back of the plume, and to steer the mm-wave beam between the two reflectors. This allows for long signal averages, while the time interval between the collection of reference and plume signals remains short.

\section{ACKNOWLEDGMENTS}

The authors thank Ronald N. Lanham and Eugene R. Koehl for their help with the field test, and Dylan Morgan for help with the data analysis. The authors also thank Mike O'Connell and Steve Schubert of 
DOE for supporting the project, Jeff Hylden of DOE for helping with test planning and coordination, Mike McLaurin of Bechtel Nevada and the entire Nevada test site staff for assisting with the tests, and Hal Goldwire and the entire Lawrence Livermore team at the test site for helping with the release profiles. The BWO power supply and the F-P cavity were built by The Ohio State University under a subcontract from Argonne National Laboratory. This work is supported by the U.S. Department of Energy, Office of Nonproliferation and National Security, Office of Research and Development, under Contract W-31-109-ENG-38.

\section{REFERENCES}

1. B. Grant, R. H. Kagann, and W. A. McClenny, "Optical remote measurement of toxic gases, J. Air Waste Manage. Assoc., vol. 42, pp. 18-30, 1992.

2. J. V. Cernius, D. A. Elser, and J. Fox, "Remote active spectrometer," SPIE Laser Applications in Metrology and Earth and Atmospheric Remote Sensing, vol. 1062, pp. 164-171, 1989.

3. W. Gordy and R. L. Cook, Microwave Molecular Spectra, John Wiley, New York, 1984.

4. N. Gopalsami, S. Bakhtiari, A. C. Raptis, S. L. Dieckman, and F. C. DeLucia, "Millimeter-wave measurements of molecular spectra with application to environmental monitoring," IEEE Trans. Instrum. Meas., vol. 45, pp. 225-230, 1996.

5. N. Gopalsami, S. Bakhtiari, A. C. Raptis, and S. L. Dieckman, "Millimeter-wave sensor for monitoring effluents," U.S. patent No. 5468964, November 1995.

6 N. Gopalsami, S. Bakhtiari, and A. C. Raptis, "Open-path millimeter-wave spectroscopy in the 225$315 \mathrm{GHz}$ range," Proc. Int. Conf. on Millimeter and Submillimeter Waves and Applications III, SPIE, vol. 2842, pp. 274-283, 1996.

7 A. Dryagin, V. V. Parshin, A. F. Krupnov, N. Gopalsami, and A. C. Raptis, "Precision broadband wavemeter for millimeter and submillimeter range," IEEE Trans. Microwave Theory Techniques, vol. 44, pp. 1610-1613, 1996.

8 L. Poynter and H. M. Pickett, "Submillimeter, Millimeter, and Microwave Spectral Line Catalog," JPL Publication 80-23, Revision 2, 1983. 